\title{
COMPOSICIÓN QUÍMICA DE TALLOS INMADUROS DE Acanthocereus spp. E Hylocereus undatus (Haw.) Britton \& Rose
}

\section{CHEMICAL COMPOSITON OF IMMATURE STEMS OF Acanthocereus spp. AND Hylocereus undatus (Haw.) Britton \& Rose}

\author{
Aída Juárez-Cruz ${ }^{1}$, Manuel Livera-Muñoz ${ }^{1 *}$, Eliseo Sosa-Montes ${ }^{2}$, Ma. Antonieta Goytia-Jiménez ${ }^{3}$, Víctor A. \\ González-Hernández ${ }^{1}$ y Ricardo Bárcena-Gama ${ }^{4}$
}

${ }^{1}$ Postgrado de Recursos Genéticos y Productividad, Fisiología Vegetal, Colegio de Postgraduados-Campus Montecillo. Km 36.5 carretera México-Texcoco. 56230, Montecillo, Texcoco, Edo. de México. ${ }^{2}$ Departamento de Zootecnia, Universidad Autónoma Chapingo. Km 38.5 carretera México-Texcoco. 56235, Chapingo, Edo. de México. ${ }^{3}$ Departamento de Preparatoria Agrícola, Universidad Autónoma Chapingo. Km 38.5 carretera México-Texcoco. 56230, Chapingo, Edo. de México. ${ }^{4}$ Postgrado de Recursos Genéticos y Productividad-Ganadería, Colegio de Postgraduados-Campus Montecillo. Km 36.5 carretera MéxicoTexcoco. 56230, Montecillo, Texcoco, Edo. de México.

${ }^{*}$ Autor para correspondencia (mlivera@colpos.mx)

\section{RESUMEN}

Los tallos inmaduros de cruceta o jacube (Acanthocereus tetragonus), nopal de cruz (A. subinermis) y pitahaya (Hylocereus undatus) son de consistencia suave y se utilizan en la gastronomía mexicana; pero hay poca información sobre su contenido nutrimental. El objetivo de este trabajo fue determinar la composición proximal y mineral de sus tallos tiernos. El material vegetal se recolectó de una plantación experimental ubicada en Tepoztlán, Estado de Morelos, México. Los resultados obtenidos se expresaron en base seca $(\mathrm{g} / 100 \mathrm{~g}$ de materia seca). El análisis proximal mostró que, en comparación con valores publicados de tallos tiernos de Opuntia spp., los tallos presentaron mayores $(\mathrm{P} \leq \mathbf{0 . 0 5})$ contenidos de proteína cruda $(11.08$ a $24.49 \mathrm{~g})$ y fibra cruda (7.86 a $14.79 \mathrm{~g})$, pero menores contenidos $(\mathrm{P} \leq$ $0.05)$ de cenizas $(10.80$ a $14.90 \mathrm{~g})$ y extracto etéreo $(0.64$ a $1.46 \mathrm{~g})$. En cuanto a minerales, las tres especies presentaron alto $(\mathrm{P} \leq \mathbf{0 . 0 5})$ contenido de $\mathrm{P}$ (0.19 a $0.37 \%), \mathrm{K}(2.19$ a $4.52 \%), \mathrm{Mg}(0.55$ a $0.66 \%)$, $\mathrm{Na}(0.07$ a $0.89 \%)$ y $\mathrm{Zn}\left(8.56\right.$ a $\left.32.15 \mathrm{mg} \mathrm{kg}^{-1}\right)$. Acanthocereus sobresalió por su alto $(\mathrm{P} \leq 0.05)$ contenido de $\mathrm{Ca}(1.51$ a $2.16 \%)$, y $\mathrm{H}$. undatus sobresalió por sus contenidos de $\mathrm{K}\left(4.82 \mathrm{mg} \mathrm{kg}^{-1}\right)$ y $\mathrm{Zn}(34.02$ $\mathrm{mg} \mathrm{kg}^{-1}$ ).

Palabras claves: Acanthocereus tetragonus, A. subinermis, Hylocereus undatus, análisis proximal, contenido de minerales.

\section{SUMMARY}

Immature stems of "cruceta or jacube" (Acanthocereus tetragonus), "nopal de cruz" (A. subinermis) and pitahaya (Hylocereus undatus) are soft, and they are used in the Mexican gastronomy. However, there is little information about their nutritional value. The objective of this work was to analyze their proximal and mineral composition in immature stems. The plant material was obtained from a plantation located in Tepoztlán, state of Morelos, México. The results were reported on dry matter basis ( $\mathrm{g} / 100 \mathrm{~g}$ of dry matter). The proximal analysis showed that compared to published values of edible young cladodes of Opuntia spp., stems presented higher ( $\mathrm{P} \leq 0.05)$ content of crude protein (11.08 to $24.49 \mathrm{~g}$ ) and crude fiber (7.86 to $14.79 \mathrm{~g})$, but lower contents $(P \leq 0.05)$ of ashes $(10.80$ to $14.90 \mathrm{~g})$ and ether extract $(0.64$ to $1.46 \mathrm{~g})$. As for minerals, the stems presented higher $(\mathrm{P} \leq 0.05)$ contents of $\mathrm{P}(0.19$ to $0.37 \%), \mathrm{K}(2.19$ to $4.52 \%), \mathrm{Mg}$ ( 0.55 to $0.66 \%), \mathrm{Na}(0.07$ to $0.89 \%)$ and $\mathrm{Zn}\left(8.56\right.$ to $\left.32.15 \mathrm{mg} \mathrm{kg}^{-1}\right)$; Acanthocereus stood out for its high $(\mathrm{P} \leq 0.05)$ Ca content $(1.51$ to $2.16 \%$ and $H$. undatus for its high $\mathrm{K}\left(4.82 \mathrm{mg} \mathrm{kg}^{-1}\right)$ and $\mathrm{Zn}(34.02 \mathrm{mg}$ $\left.\mathrm{kg}^{-1}\right)$ content.

Index words: Acanthocereus tetragonus, A. subinermis, Hylocereus undatus, mineral content, proximal analysis.

\section{INTRODUCCIÓN}

Algunas cactáceas son parte de la dieta humana desde hace 9000 años y otra de sus ventajas es su adaptación a regiones de baja precipitación por tener tolerancia a la sequía (Nobel, 1998). Las cactáceas también han jugado un papel ecológico importante en frenar la degradación de suelos deforestados (Sáenz, 2006a). De los cactos cultivados en regiones áridas y semiáridas, uno de los más estudiados es el nopal (Opuntia spp.), por su importancia en la alimentación humana y animal tanto de sus frutos como de sus cladodios tiernos conocidos como "nopalitos". El sabor característico y la versatilidad para 
prepararlos son algunas de las razones por la que se utilizan en platillos de la gastronomía mexicana e internacional. En lo que respecta a su contenido nutrimental, el nopal contiene elevadas cantidades de fibra, calcio, magnesio, hierro y sodio (Bravo, 1978; Sáenz, 2006b), compuestos necesarios para la salud humana.

Entre las especies que toleran la sequía y poseen cualidades nutrimentales que benefician al ser humano, están la cruceta o jacube (Acanthocereus tetragonus), el nopal de cruz (A. subinermis) y la pitahaya (Hylocereus $s p$ ), cuyas plantas y órganos se destinan a diferentes usos como: cercos vivos, ornamentales, medicinales $y$ alimenticios. La importancia de A. tetragonus y A. subinermis radica en el uso de los tallos como verdura para la elaboración de numerosos guisos (Juárez-Cruz, 2007; Com. pers. ${ }^{1}$ ). La planta de pitahaya se puede aprovechar íntegramente, y si bien ha destacado por la importancia económica de sus frutos (Livera et al., 2010), sus tallos inmaduros también se utilizan para consumo humano (Castillo et al., 2005). Según Córdoba et al. (2000), A. tetragonus contiene $5 \mathrm{~g}$ de proteína cruda (PC) y $16.3 \mathrm{~g}$ de fibra cruda (FC) por cada $100 \mathrm{~g}$ de materia seca (MS); y $4.68 \mathrm{mg} \mathrm{kg}{ }^{-1}$ de calcio en base seca. En H. undatus, Rodríguez (1997) reportó $14.84 \mathrm{~g}$ de PC, $21.50 \mathrm{~g}$ de FC y $39.94 \mathrm{~g}$ de ELN.

Hasta ahora los análisis proximal y mineral eran incompletos para estas especies y para A. subinermis no había información al respecto, por lo que el objetivo de este estudio fue analizar la composición proximal y mineral de tallos tiernos de A. tetragonus, A. subinermis $y$ $H$. undatus, cultivados bajo las mismas condiciones ambientales y de manejo, con la finalidad de contribuir al conocimiento del potencial alimenticio de las especies mencionadas, y así apoyar la promoción de su cultivo en el trópico seco.

\section{MATERIALES Y MÉTODOS}

\section{Ubicación de la plantación}

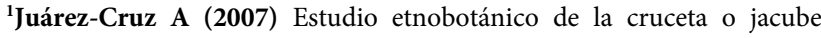
(Acanthocereus spp.) en la zona centro de Veracruz. Tesis Profesional. Universidad Autónoma Chapingo. Chapingo, Texcoco, Estado de México. 155 p.
}

La plantación se ubica en Tepoztlán, Morelos ( $18^{\circ} 55^{\prime}$ LN, 99 07’ LO), a una altitud de $1732 \mathrm{~m}$; su clima es cálido $\operatorname{Awo}(w), \operatorname{Aw} 1(w)$ con temperatura media anual entre 22 y $26^{\circ} \mathrm{C}$, con lluvias en verano y con invierno seco (García, 1988). El suelo es franco-arcilloso con un $\mathrm{pH}$ de 7.81, materia orgánica de $2.95 \%$ y una fertilidad (en mg $\mathrm{kg}^{-1}$ de suelo) de 153 de fósforo, 400 de potasio, $8538 \mathrm{de}$ calcio y 1936 de magnesio. Se le aplicaron riegos de auxilio con solución nutritiva Steiner a $100 \%$, cada 15 d durante los meses de sequía (de noviembre a abril).

\section{Material vegetal}

De plantas de 6 meses de edad establecidas en campo, se obtuvieron tallos tiernos de cruceta o jacube (Acanthocereus tetragonus), nopal de cruz (A. subinermis) y pitahaya (Hylocereus undatus, genotipos CP-171 y CP182), que se recolectaron en forma continua de otoño de 2009 a verano de 2010, cuando tenían de 15 a $25 \mathrm{~cm}$ de longitud y de 20 a $30 \mathrm{~d}$ de edad. Las muestras se depositaron en bolsas de polietileno y se trasladaron al laboratorio a temperatura ambiente $\left(20 \pm 2{ }^{\circ} \mathrm{C}\right)$.

En el laboratorio los tallos se lavaron con agua destilada y secaron con papel absorbente; después se les retiraron las areolas y en algunos casos como en $A$. tetragonus y A. subinermis se les retiró parte de la cutícula. Cada material se cortó en fracciones de aproximadamente $1 \mathrm{x} 1 \mathrm{~cm}$, las cuales se secaron en una estufa de aire forzado a $50{ }^{\circ} \mathrm{C}$ hasta peso constante, de acuerdo con el método establecido por la Association of Official Analytical Chemists (AOAC, 1990). El material seco se trituró con un molino eléctrico (Modelo $\mathrm{Kb}$ 5/10 Janke and Kunkel IKA®, Germany) provisto con criba de $1 \mathrm{~mm}$, hasta obtener más de $50 \mathrm{~g}$, cantidad suficiente para los análisis. Cada muestra se guardó en un recipiente de plástico a temperatura ambiente $\mathrm{y}$ en oscuridad. Finalmente, se obtuvo una muestra compuesta de cada material.

\section{Análisis proximal y de minerales}

El análisis proximal de cada muestra consistió en la determinación de humedad, cenizas, materia seca (MS), extracto etéreo (EE), fibra cruda (FC), proteína cruda (PC) y extracto libre de nitrógeno (ELN), de acuerdo con los métodos establecidos por la AOAC (1990). El análisis de minerales consistió en la determinación de fósforo $(\mathrm{P})$ por 
colorimetría, según el método del molibdato-vanadato; potasio $(\mathrm{K})$, sodio $(\mathrm{Na})$, calcio $(\mathrm{Ca})$, magnesio $(\mathrm{Mg})$, hierro $(\mathrm{Fe})$, manganeso $(\mathrm{Mn})$, cobre $(\mathrm{Cu})$ y zinc $(\mathrm{Zn})$ se determinaron por espectrofotometría de emisión atómica ICP-OES Varian ${ }^{\circledR}$ Liberty Series II Springvale, Australia. Los resultados de composición química se expresaron en base seca (g/100 g MS).

\section{Análisis estadístico}

A los datos obtenidos del análisis proximal se les aplicó un análisis de varianza bajo un diseño experimental completamente al azar y una prueba de comparación de medias (Tukey, 0.05), ambos con el programa SAS 1999 versión 8.0. En el análisis proximal se reportan los promedios y sus desviaciones estándar de tres repeticiones; en el análisis de minerales no hubo repeticiones.

\section{RESULTADOS Y DISCUSIÓN}

\section{Análisis proximal}

El análisis de varianza mostró que no hubo diferencias entre las especies $(\mathrm{P} \leq 0.05)$ para el contenido de humedad, pero sí las hubo para el resto de las variables. $A$. tetragonus $y$ A. subinermis presentaron mayor $(\mathrm{P} \leq 0.05)$ contenido de proteína y de fibra, y menor $(\mathrm{P} \leq 0.05)$ contenido de extracto libre de $\mathrm{N}$ en comparación con $H$. undatus, lo que puede indicar una mayor calidad nutritiva del género Acanthocereus (Cuadro 1). Los resultados aquí obtenidos son superiores a los reportados por Córdoba et al. (2000) para A. tetragonus, pero menores a los reportados por Rodríguez (1997) en $H$. undatus; lo cual puede atribuirse a las diferencias genotípicas y ambientales en las que se manejaron las especies en cada estudio (Omidi et al., 2010).

Al comparar los resultados del análisis proximal de $A$. tetragonus, A. subinermis y $H$. undatus con los encontrados en cladodios tiernos de nopal (Opuntia spp.) por Stintzing y Carle (2005), Guzmán y Chávez (2007), Rodríguez-García et al. (2007), Ramírez-Tobías et al. (2007) y Hernández-Urbiola et al. (2010), se encontró que en todos los casos la composición proximal fue similar, lo que sugiere que estos tres materiales estudiados son similares en sus cualidades nutrimentales y que los tres constituyen una alternativa alimenticia.

\section{Composición mineral}

Los minerales tienen numerosas funciones en el organismo humano, como mantener la presión osmótica, formar parte de la estructura de muchos tejidos, ser constituyentes esenciales de ciertas hormonas y regular los latidos cardíacos (Latham, 2002). En este caso, A. tetragonus, A. subinermis y H. undatus (CP-171y CP-182) tuvieron altos contenidos de $\mathrm{P}, \mathrm{K}, \mathrm{Mg}, \mathrm{Na}$ y $\mathrm{Cu}$. Las especies A. tetragonus y A. subinermis destacan por su elevado contenido en $\mathrm{Ca}$; los genotipos de $H$. undatus (CP-171y CP-182) sobresalieron por su contenido de $\mathrm{K}$ y Zn, respectivamente (Cuadro 2).

Cuadro 1. Análisis proximal de tallos inmaduros de Acanthocereus spp. e Hylocereus sp. expresados en base seca (g/100 g).

\begin{tabular}{|c|c|c|c|c|}
\hline Nutrimento & $\begin{array}{l}\text { A. tetragonus } \\
\qquad \mathrm{x} \pm \mathrm{ds}\end{array}$ & A subinermis & $\begin{array}{c}\text { H. undatus } \\
\text { CP-171 } \\
\mathrm{X} \pm \mathrm{ds}\end{array}$ & $\begin{array}{c}\text { H. undatus } \\
\text { CP-182 } \\
\mathrm{X} \pm \mathrm{ds}\end{array}$ \\
\hline Humedad total & $87.95 \pm 2.55 \mathrm{a}$ & $89.31 \pm 3.37 \mathrm{a}$ & $91.30 \pm 2.40 \mathrm{a}$ & $90.53 \pm 2.60 \mathrm{a}$ \\
\hline Cenizas & $12.12 \pm 0.09 \mathrm{~b}$ & $14.90 \pm 0.06 \mathrm{a}$ & $12.36 \pm 0.18 b$ & $10.80 \pm 0.09 c$ \\
\hline Proteína cruda & $24.49 \pm 1.80 \mathrm{a}$ & $21.77 \pm 0.26 \mathrm{a}$ & $11.08 \pm 0.50 \mathrm{~b}$ & $12.15 \pm 1.12 b$ \\
\hline Fibra cruda & $15.80 \pm 0.14 \mathrm{a}$ & $13.38 \pm 0.90 b$ & $8.11 \pm 0.15 c$ & $7.86 \pm 0.95 c$ \\
\hline Extracto etéreo & $1.23 \pm 0.04 \mathrm{ab}$ & $0.64 \pm 0.06 \mathrm{~b}$ & $0.94 \pm 0.15 \mathrm{ab}$ & $1.46 \pm 0.48 \mathrm{a}$ \\
\hline ELN & $46.35 \pm 0.43 b$ & $49.31 \pm 0.15 b$ & $67.51 \pm 0.68 \mathrm{a}$ & $67.72 \pm 0.45 \mathrm{a}$ \\
\hline
\end{tabular}

x: promedios de tres repeticiones; ds: desviación estándar. Medias con letras distintas en una misma fila, son estadísticamente diferentes (Tukey, 0.05). ELN = Extracto libre de nitrógeno. 
Cuadro 2. Composición mineral de tallos inmaduros de Acanthocereus spp. e Hylocereus sp. (base seca).

\begin{tabular}{ccccc}
\hline Componente & A. tetragonus & A. subinermis & $\begin{array}{c}\text { H. undatus } \\
\text { CP-171 }\end{array}$ & $\begin{array}{c}\text { H. undatus } \\
\text { CP-182 }\end{array}$ \\
\hline $\mathrm{P}(\%)$ & 0.35 & 0.40 & 0.20 & 0.24 \\
$\mathrm{~K}(\%)$ & 2.80 & 2.42 & 4.82 & 2.31 \\
$\mathrm{Ca}(\%)$ & 2.31 & 1.62 & 0.43 & 0.48 \\
$\mathrm{Mg}(\%)$ & 0.65 & 0.62 & 0.70 & 0.58 \\
$\mathrm{Na} \mathrm{( \% )}$ & 0.17 & 0.95 & 0.10 & 0.07 \\
$\mathrm{Cu}\left(\mathrm{mg} \mathrm{kg}^{-1}\right)$ & 5.86 & 6.35 & 8.99 & 16.00 \\
$\mathrm{Fe}\left(\mathrm{mg} \mathrm{kg}^{-1}\right)$ & 28.16 & 22.80 & 7.53 & 28.80 \\
$\mathrm{Mn}\left(\mathrm{mg} \mathrm{kg}^{-1}\right)$ & 118.24 & 50.61 & 30.48 & 40.01 \\
$\mathrm{Zn}\left(\mathrm{mg} \mathrm{kg}^{-1}\right)$ & 29.18 & 25.76 & 9.12 & 34.02 \\
\hline
\end{tabular}

Los niveles de $\mathrm{P}, \mathrm{K}, \mathrm{Mg}$, Na, Mn y Zn obtenidos para A. tetragonus son superiores a los reportados por Córdoba et al. (2000), contraste que puede deberse a diferencias entre poblaciones y en sus condiciones de manejo (Omidi et al., 2010). El contenido mineral en A. tetragonus, A. subinermis, $H$. undatus $\mathrm{CP}-171$ y CP-182 fue similar a los reportados en nopal (Opuntia sp.) por Córdoba et al. (2000), Rodríguez-García et al. (2007), HernándezUrbiola et al. (2010) y Stintzing y Carle (2005). Esto indica que además de proteínas, grasas y otros componentes, los tres cactos aquí evaluados también aportan minerales necesarios para el organismo. Las diferencias observadas en la composición proximal y mineral de los tallos tiernos entre las especies evaluadas, pueden deberse al genotipo, a factores ambientales, a la estación de crecimiento y la edad de la planta (Stintzing y Carle, 2005; Omidi et al., 2010; Contreras-Padilla et al., 2011).

En conclusión, los tallos de A. tetragonus, A. subinermis y $H$. undatus, tienen altos contenidos de humedad, proteína cruda y fibra cruda, y de minerales esenciales para el ser humano como $\mathrm{P}, \mathrm{K}, \mathrm{Mg}, \mathrm{Na}$ y $\mathrm{Cu}$. Tales características nutricionales permiten la oportunidad de promover el uso y cultivo de estas especies estudiadas, y servir de punto de partida para futuras investigaciones en ellas.

\section{BIBLIOGRAFÍA}

AOAC Association of Official Analytical Chemists (1990) Official Methods of Analysis. 15th ed. Association of Official Analytical Chemists. Washington, DC, USA. 1298 p.

Bravo H H (1978) Las Cactáceas de México. Tomo 1. Ed. Universidad Nacional Autónoma de México. México. 743 p.
Castillo M R, M Livera M, G J Márquez G. (2005) Caracterización morfológica y compatibilidad sexual de cinco genotipos de pitahaya (Hylocereus undatus). Agrociencia 39:183-194.

Contreras-Padilla M, E Pérez-Torrero, Margarita I. HernándezUrbiola, G Hernández-Quevedo, A del Real, E M RiveraMuñoz, M E Rodríguez-García (2011) Evaluation of oxalates and calcium in nopal pads (Opuntia ficus-indica var. Redonda) at different maturity stages. J. Food Comp. Anal. 24:38-43.

Córdoba L M, M M Hernández-Martínez, E Sánchez-Martínez (2000) Contribution to the present knowledge of Acanthocereus tetragonus (Linnaeus) Hummelink from the Sierra Gorda of Querétaro. Cact. Suc. Mex. 45:34-39.

García E (1988) Modificaciones al Sistema de Clasificación Climática de Köppen. 4a. ed. Offset Larios. México, 271 p.

Guzmán L D, J Chávez (2007) Estudio bromatológico del cladodio del nopal (Opuntia ficus-indica) para el consumo humano. Rev. Soc. Quím. Perú. 73:41-45.

Hernández-Urbiola M I, M Contreras-Padilla, E Pérez-Torrero, G Hernández-Quevedo, J I Rojas-Molina, M E Cortes, M E Rodríguez-García (2010) Study of nutritional composition of Nopal (Opuntia ficus indica cv. Redonda) at different maturity stages. Open Nutr. J. 4:11-16.

Latham M C (2002) Nutrición Humana en el Mundo en Desarrollo. Colección FAO: Alimentación y nutrición No. 29. 531 p.

Livera M M, Y D Ortiz H, R Castillo M, F Castillo G, R Martínez C, J J Ramírez D, A J Valencia B, J A Carrillo S (2010) Pitahaya (Hylocereus spp.): problemas, logros y perspectivas. In: S Cruz I, A Muratalla L, A T Kato Y (comps). La Investigación al Servicio del Campo Mexicano. Ed. Postgrado en Recursos Genéticos y Productividad-Genética. Colegio de Postgraduados. Montecillo, Texcoco, Edo. de México. pp:57-59.

Nobel P S (1998) Los Incomparables Agaves y Cactos. Ed. Trillas. México. $211 \mathrm{p}$.

Omidi H, Z Tahmasebi, H A Naghdi B, H Torabi, M Miransari (2010) Fatty acid composition of canola (Brassica napus L.), as affected by agronomical, genotypic and environmental parameters. C. R. Biologies 333:248-254.

Ramírez-Tobías H M, J A Reyes A, J M Pinos R, J R Aguirre R (2007) Efecto de la especie y madurez sobre el contenido de nutrientes de cladodios de nopal. Agrociencia 41:619-626. 
Rodríguez C A (1997). Guía técnica para la producción de plantas de pitahaya en viveros. Maxcanú, Yucatán, Secretaría de Desarrollo Social y Universidad Autónoma Chapingo. México. 70 p.

Rodríguez-García M E, C de Lira, E Hernández-Becerra, M A Cornejo-Villegas, A J Palacios-Fonseca, I Rojas-Molina, R Reynoso, L C Quintero, A del Real, T A Zepeda, C MuñozTorres (2007) Physicochemical characterization of nopal pads (Opuntia ficus indica) and dry vacuum nopal powders as a function of the maturation. Plant Foods Hum. Nutr. 62:107-112.
Sáenz C (2006a) Los nopales como recurso natural. In: Utilización Agroindustrial del Nopal. C. Rosell (ed). FAO, Boletín de Servicios Agrícolas 162. pp:1-6.

Sáenz C (2006b) Características y composición química de los nopales. In: Utilización Agroindustrial del Nopal. C. Rosell (ed). FAO, Boletín de Servicios Agrícolas 162. pp:7-22.

Stintzing F C, R Carle (2005) Cactus stems (Opuntia spp.): A review on their chemistry, technology, and uses. Mol. Nutr. Food Res. 49:175-194. 